\title{
PETRÓLEO, MEDIO AMBIENTE, CAMBIO CLIMÁTICO Y SEGURIDAD: MACONDO, OTRA ADVERTENCIA MÁS
}

\author{
Gian Carlo Delgado Ramos ${ }^{1}$ \\ Universidad Nacional Autónoma de México
}

\begin{abstract}
Resumen.- El actual patrón energético está centrado esencialmente en combustibles fósiles, siendo el petróleo el más relevante. El proceso de su obtención y quema produce costos ambientales y humanos que no son tomados en cuenta y por tanto quedan ocultos. Uno de tantos son los derrames, como el sucedido en el pozo Macondo en abril de 2010. El presente texto plantea que tales costos ocultos deben leerse desde un análisis amplio que no se limite al suceso per se. De este modo se plantea en un primer momento, la necesidad de dar cuenta del eminente alcance del punto máximo de producción (peak oil), el esperado incremento de la población mundial y el creciente calentamiento del planeta. Se analiza entonces el significado del derrame del pozo Macondo como un rasgo que lejos de ser excepcional es característico del patrón energético actual. Se cierra con una valoración sobre las implicaciones de seguridad del actual patrón energético para luego plantear la necesidad de repensar el desarrollo como sustento de un cambio de paradigma.
\end{abstract}

Palabras clave.- petróleo, energía, derrames petroleros, costos ocultos, seguridad, cambio climático.

Summary.- Current energy paradigm is essentially based on fossil fuels, mainly oil. The process of its extraction, transformation and consumption produces environmental and human costs that are usually not internalized. As such they can be categorized as hidden costs of the current energy pattern. Spills, such as the one of Macondo's well that took place on April 2010, are among those hidden cost. This article considers that such costs should be analysed from a wider perspective, avoiding focalized readings in the sense of limiting evaluations to the impacts of each case. From such viewpoint the significance of Macondo oil spill is described, not as an exception, but as a constant fact within the current energy pattern. At the same time it is considered necessary to take into account at least three variables in our reading: peak oil, the expected increase of world population and climate change. Considering the latest, the text offers an appraisal of the energy paradigm's security implications, concluding with a consideration on the necessity of rethinking development as starting point for a real and profound change of paradigm.

Key words.- oil, energy, oil spills, hidden costs, security, climate change.

\section{Introducción}

Cuando la humanidad tuvo acceso a fuentes altamente condensadas de energía, su expansión y complejidad tuvo lugar como nunca antes. La escala global de tal fenómeno incluyó lo espacial, lo poblacional y desde luego, lo energético. Se pasó de un consumo de unos miles de barriles de crudo al año a mediados del siglo XIX a más de 65 millones de barriles diarios para fines del siglo XX (Heinberg, 2003: 92). Mientras más energía se dispuso, más espacio se ocupaba, siendo la ciudad ícono de ése proceso. El crecimiento poblacional

\footnotetext{
${ }^{1}$ Economista egresado de la Universidad Nacional Autónoma de México (UNAM). Doctor en "Ciencias Ambientales" por la Universidad Autónoma de Barcelona, España. Es investigador de tiempo completo del Centro de Investigaciones Interdisciplinarias en Ciencias y Humanidades de la UNAM. Integrante del Sistema Nacional de Investigadores del Consejo Nacional de Ciencia y Tecnología de México.
} 
se disparó, especialmente a partir de la segunda mitad del siglo XX pues pasó de unos 500 millones hasta el siglo XVI, a mil millones a principios del siglo XIX y dos mil millones para la década de 1930, para después aumentar en un mil millones adicionales de personas para 1960, 1974, 1987 y 1999 (HomerDixon, 2007: 61). Hoy día, la población mundial se estima en unos 6,800 millones de habitantes, siendo poco más de la mitad urbana.

Los combustibles fósiles constituyen, según la Agencia Internacional de Energía (AIE) y para el año 2008 , el $81.3 \%$ de la energía primaria total mundial (AIE, 2010: 6). De este porcentaje, la mayor parte del petróleo se emplea en motores de combustión interna destinados al transporte, el resto en generación de electricidad y en la petroquímica. La mitad del carbón se emplea en la generación de energía eléctrica y el resto en diversas funciones industriales y domésticas. El gas, se emplea crecientemente en la generación de electricidad pues se pasó del $12.1 \%$ de su uso en el total de energía eléctrica generada en 1973 a 21.3\% en 2008. También se usa, y prácticamente en montos similares, por la industria, el comercio y usos domésticos. De precisar es que la mayoría de la electricidad, o energía secundaría, se emplea en usos industriales (54\%) y doméstico-comerciales (46\%).

Lo indicado implica que, en resumen, el destino general de los combustibles fósiles tiene tres grandes rutas: 1) generación de energía calórica; 2) de energía eléctrica; y 3) motores de combustión interna. Es un contexto en el que resulta imperante notar que de 1973 a 2008, si bien la cantidad de energía generada se ha duplicado -se pasó de 6,115 a 12,267 millones de toneladas de petróleo equivalente-, la proporción de los combustibles fósiles no ha variado aunque sí se le da un mayor peso al carbón y al gas. A ello se suma un incremento en el rol de la energía nuclear que creció seis veces al tiempo que, llamativamente, se estancan las energías renovables al representar en esos 35 años tan sólo el $10 \%$ del total de energía primaria mundial (AIE, 2010). Así, los datos nos muestran que, pese a las adversidades, la política energética de las últimas décadas ha sido marcada y claramente fósil y nuclear.

Dejando de lado el caso de la nuclear (véase Delgado, 2008 para una indagación puntual) y enfocándonos en el caso de los fósiles, es importante recordar que en especial el ritmo de extracción y quema de petróleo ha llevado a que estemos ya en su punto máximo de producción (o de peak oil). Marion King Hubbert estimó que el pico mundial se alcanzaría entre 1990 y 2000, sin embargo muchos de los datos de pozos petroleros que empleó no eran del todo precisos, además de que, desde entonces, la tecnología de extracción posibilitó ampliar ligeramente las reservas probadas de crudo. Colin J. Cambell (1997), otro geólogo petrolero, actualizó la estimación y fijó el "pico" mundial entre el 2008 y 2010. En el mismo sentido, Kenneth Deffeyes (2001) habla de un pico de entre 2003 a 2009, mientras que L. F. Ivanhoe, fundador del Hubbert Center for Petroleum Supply Studies, coincide en que el pico se alcanzó entre el 2000 y el 2010. Otros, como el geólogo Thomas Magoon del US Geology Survey (USGS) o el Oil \& Gas Journal, son relativamente más optimistas y hablan de un rango de años de entre el 2003 y el 2020 (Heinberg, 2003: 113). Pero, como bien advierte Homer-Dixon, la situación podría ser peor que la estimada puesto que los datos de las reservas mundiales (tanto de las 
petroleras privadas como públicas) usualmente son inexactas e incluso deliberadamente manipuladas en tanto que permiten estimular la economía nacional, abrir las puertas a más créditos y, en el caso de los países miembros de la Organización de Países Exportadores de Petróleo (OPEP), adjudicarse mayores cuotas de producción (Homer-Dixon, 2007: 89).

A lo anterior, deben sumarse las estimaciones de la AIE que contemplan un aumento en el consumo energético del 57\% en el periodo de 2004 a 2030, un panorama en el que no es menor el hecho de que el consumo energético sea desigual pues se calcula que los habitantes de los países con mayores ingresos consumen unas 21 veces per capita más que los de bajos ingresos. Cifras a nivel mundial precisan, además, que 2,400 millones de personas utilizan biomasa tradicional (e.g., madera) para cocinar, mientras que 1,600 millones no tienen acceso a la electricidad (Bank Information Center et al, 2006: 21). Esto es: la mitad de la población mundial está prácticamente fuera de los supuestos "beneficios de la modernidad". Por tanto, cuando se habla de patrones intensivos de consumo energético, en buena medida nos referimos al de una fracción de la población mundial, esto es el de las clases medias y altas.

\section{Los costos ocultos del patrón energético: una breve mirada al caso del petróleo.}

Uno de los argumentos de mayor peso para abogar a favor del patrón energético fósil de cara al desarrollo de energías "sustentables" ${ }^{2}$ es que el petróleo, carbón y gas siguen siendo las fuentes de energía más baratas. Se trata de una afirmación que sin embargo se sustenta en una muy peculiar contabilidad.

Independientemente de que estamos hablando de un tipo de energía limitada la energía fósil se presenta como stock y no como flujo tal y como sí lo es la energía solar-, lo que es un hecho es que en el costo, no sólo de producción de la energía fósil, sino del mantenimiento del patrón energético fósil in toto, hay un amplio abanico de "externalidades ocultas" que no son tomadas en cuenta $y$, que de hacerse, sin duda lo tornarían costoso no sólo en términos económicos, sino también socio-ecológicos. A ello debería sumarse, en negativo, los subsidios otorgados (unos 200 mil mdd al año (Godrej, 2001: 134)) y los costos de seguridad ejercidos para garantizar y mantener el fluido curso de combustibles fósiles hacia los mayores consumidores, un costo que se estima en al menos el $25 \%$ del gasto total en defensa a nivel mundial (Steffes, 1994: 20-29).

Los mencionados costos ocultos se pueden identificar en todo el proceso producción-circulación-consumo. Dígase para el caso del petróleo, desde la

\footnotetext{
${ }^{2}$ No debe considerarse como "energía sustentable" a la nuclear como así ha procurado que se haga la propia industria nucleoeléctrica. Para un análisis al respecto y mayores referencias, léase Delgado, 2008.
} 
exploración, perforación y extracción, hasta la transportación, refinación y quema.

Tan sólo para mostrar algunos aspectos relevantes vale indicar que en la exploración no se toma en cuenta la alteración de los ecosistemas inmediatos a la zonas de excavación, tanto por el movimiento de equipo y maquinaria, como por las propias explosiones. El impacto es considerable puesto que una vez identificadas las zonas petroleras potenciales, es necesario comprobar su existencia mediante la perforación de pozos de prueba. Una vez encontrado el combustible, las perforaciones se amplían de entre 10 a 30 pozos por plataforma petrolera con un rango de fallo del 40 por ciento(Esptein y Selber, 2002: 9).

En la perforación se utilizan una gran cantidad de explosivos, incluso se han llegado a utilizar cargas nucleares controladas (caso de la entonces URSS) (Ibid). Tales técnicas de perforación y el posterior emplazamiento de plataformas petroleras contamina, altera y fragmenta los ecosistemas. Aún más, con la extracción de petróleo, se sabe que es común encontrar depósitos subterráneos de materiales radioactivos en estado natural. La frecuencia en la que ésos son removidos y traídos a la superficie y el escaso monitoreo de ello aún en comparación con otras actividades como la minera no energética- ha llevado a advertir que los riesgos pueden ser considerablemente altos puesto que inclusive bajos niveles de radiación pueden tener impactos mutagénicos en la biodiversidad (Ibid: 11).

En la extracción de petróleo, entre otros impactos, debe considerarse el uso masivo de agua y los cuantiosos desechos producidos de impacto ecológico diverso puesto que contienen metales pesados, compuestos tóxicos como el mercurio e hidrocarburos aromáticos volátiles (benceno, tolueno y exileno, con capacidad de imitar las hormonas y por tanto de deteriorar el desarrollo y reproducción de animales y del ser humano), entre otros. En promedio, se estima que en la producción petrolera terrestre, los desechos de lodos van desde los 270 mil litros a poco menos del millón y medio de litros diarios. En las plataformas marinas el agua de desecho ronda casi los 2 millones de litros diarios (Ibid: 25). Así, mientras los lodos son usualmente vertidos a la tierra (con un tratamiento parcial de las aguas de desecho), las aguas de las plataformas marinas son casi en su totalidad derramadas directamente a los océanos. Reservas de agua subterránea y superficial, así como la biodiversidad, se ven afectadas por tales desechos en tierra, al tiempo que las aguas de desecho en los océanos pueden ser arrastradas por las corrientes marinas a cientos de kilómetros de distancia afectando con ello los ecosistemas que encuentren a su paso.

A lo anterior se suman los riesgos de explosión, derrames e incendios provocados como parte del funcionamiento cotidiano de los pozos petroleros, por la transferencia del crudo de una instalación a otra, por error humano, etcétera.

Igualmente, deben contabilizarse los gases de efecto invernadero y otros contaminantes atmosféricos que genera la extracción, el transporte y la 
refinación del crudo. Tan sólo la quema del gas natural asociado al proceso de extracción, práctica barata y muy común, se estima en el orden de unos 35 millones de toneladas de dióxido de carbono y 12 millones de toneladas de metano (Ibid). Asimismo, la extracción y transportación implica el permanente riesgo de derrames de diversa envergadura y de diverso impacto socioambiental. Ese riesgo no es sin embargo una eventualidad de la industria, por el contrario se hace constantemente presente.

Los derrames de gran escala, de más de 10 millones de galones, han ocurrido prácticamente cada año desde la década de 1960, mientras que los derrames de menor escala, aunque Ilaman menos la atención pública, de hecho se calcula que en conjunto suman una cantidad mucho mayor de petróleo vertido que el de los grandes derrames (Ibid: 20 -21). Los impactos son enormes (véase más adelante), más en ecosistemas acuáticos dada la menor densidad del petróleo con respecto al agua. Así, una tonelada de crudo derramada típicamente cubre unos $12 \mathrm{~km}^{2}$ de agua (Ibid: 22 ).

Llegando al usuario final, la quema de petróleo genera una serie de contaminantes que, como es de conocimiento público, ha contribuido de manera primaria en el calentamiento global del planeta. Seis son los principales elementos que contribuyen a la contaminación del aire: compuestos orgánicos volátiles (generados por la combustión de combustibles fósiles); dióxido de sulfuro (producido por la quema de carbón); dióxido de carbono; partículas de 10 micrones o menos (humo, polvo, vapores, etcétera, producto sobre todo de la quema del diesel); partículas de 2.5 micrones o menos o PM-2.5s, similares a las anteriores pero de mayor daño a la salud humana (sobre todo a los tejidos pulmonares); y aditivos de tetraetíl usualmente empleados para mejorar la eficiencia de la gasolina como combustible.

Los impactos de corto-mediano plazo de tales contaminantes o smog incluyen la contaminación de la vegetación, la filtración de contaminantes a los mantos acuíferos y de ahí al resto de la cadena alimenticia, lluvia ácida, enfermedades diversas como asma, problemas cardiovasculares, cáncer, irritación y alergias, etcétera. Los impactos de largo plazo o "indirectos" están esencialmente vinculados con las implicaciones del calentamiento global tal y como ha sido ampliamente evaluado y descrito en los informes del IPCC (www.ipcc.ch). Véase más adelante.

\subsection{El derrame del pozo Macondo en el Golfo de México.}

Los derrames en la industria petrolera, como ya se precisó, lejos de ser ocasionales, son sistemáticos. El caso del pozo Macondo es sólo de los más recientes (mega)desastres socio-ambientales producidos por la industria petrolera, antecedido por otros de tal orden como el del Prestige en España (2002), los asociados a la guerra del Golfo Pérsico en Kuwait (1991), el accidente del Exxon Valdez en Alaska (1989), el caso Ixtoc I en México (1979), el de Amoco Cadiz en Bretaña, Francia (1978), etcétera. 
En el caso del derrame del pozo Macondo, una perforación de exploración parte de una plataforma marina operando a 2,400 metros de profundidad y perforando al momento de la explosión a unos $1,500 \mathrm{~m}$, la cantidad de petróleo vertido pasó de unos 800 barriles diarios a unos 25 mil barriles diarios El total del derrame que se prolongó del 20 de abril al 5 de agosto de 2010 se estimó por parte del grupo técnico de expertos (FRTG) en unos 4.9 millones de barriles o una y media veces el accidente de Ixtoc I en México (www.doi.gov/deepwaterhorizon/loader.cfm?csModule=security/getfile\&PageID $=33972$ ).

La operación petrolera de aguas profundas, del tipo al que apuesta hoy día el gobierno mexicano en aguas nacionales, fue claramente de alto riesgo y de minimización de potenciales impactos ya conocidos dado los accidentes previos, en 2001 el de la plataforma P-36 en Brasil a 130 km de la costa de Río de Janeiro y en 2009 el de la plataforma West Atlas en el mar de Timor. Los riesgos eran pues elevados no sólo por la profundidad y las presiones que implicaba su operación, sino por que se trataba de una zona de alta frecuencia de huracanes y fenómenos meteorológicos tropicales y porque además, se encontraba relativamente próxima a un área de diversidad biológica marina y costera de relevancia.

La ubicación del proyecto petrolero a la plataforma continental de EUA, a unos $66 \mathrm{~km}$ de distancia de la costa de Louisiana, fue un factor que potenció la afectación socio-ambiental y la visibilidad del derrame puesto que se extendió rápidamente a lo largo de la costa de Louisiana, Missisipi, Alabama y parte de Florida (al menos hasta Panama City). ${ }^{3}$ Además, se expandió aguas adentro.

Para "manejar" el derrame, se capturó una fracción (17\%), se quemó (8\%) y se dispersó químicamente otra (8\%). Para este último procedimiento, claramente de ocultamiento parcial del impacto, British Petroleum (BP) definió emplear el dispersante Corexit 9500 y 9527. ${ }^{4}$ Si bien la mancha ya no fue superficialmente detectable desde agosto de 2010, el petróleo sigue ahí pues se estima que ése permanece por un tiempo suspendido en pequeños glóbulos (de ser ingerido en esta forma se puede bioacumular en los tejidos de los animales con afectaciones diversas) para luego depositarse en el lecho marino con consecuencias aún en buena parte desconocidas, no sólo por la presencia del crudo en sí misma, sino también porque el químico empleado crea un entorno tóxico con efectos mortales para especies sensibles y/o potenciales afectaciones cancerígenas en otras. Desde luego, los impactos dependerán del grado de exposición de las especies tanto al petróleo como al dispersante, las relaciones de interdependencia y capacidad de movilidad de las mismas, pero, los ecosistemas en cuanto tales, tardarán decenas de años en recuperarse, si es que eso es posible en su totalidad.

\footnotetext{
${ }^{3}$ Para una modelación del derrame, véase:

www.nytimes.com/interactive/2010/05/01/us/20100501-oil-spill-tracker.html

${ }^{4}$ El Corexit 9500 contiene sorvitan, ácido butanodioico y destilados del petróleo. El Corexit 9527 es producido con 2-butoxiletanol y un compuesto orgánico de baja concentración de propilenglicol.
} 
Es pues en este tenor llamativo que los dispersantes empleados hayan sido compuestos, estrictamente hablando, de fase experimental pues la propia empresa fabricante (Nalco Holdings, filial de BP) reconoce que no se han realizado estudios de toxicidad -pese a ello se asegura que el potencial de daños a la salud humana es moderado 0 bajo (www.Imrk.org/corexit 9500 uscueg.539287.pd www.doh.state.fl.us/chd/bay/Documents/Oilspill/Master EC9527A MSDS 5392 95.pdf). Aún así y con tales antecedentes se empleo en cantidades indiscriminadas pues representaba no la mejor opción, sino la más económica. De ese modo, entre 7 y 8 millones de litros de Corexit fueron vertidos al Golfo (poco más de la mitad de modo superficial y el resto inyectado debajo del agua).

La acción, en efecto, ha permitido mantener los impactos ambientales imperceptibles, al menos por el momento, pero no significa que no existan. Preocupan especialmente aquellos impactos en evolución que se expresarán eventualmente en el mediano y largo plazo y que por esa misma razón serán difíciles de asociar al desastre de Macondo. De considerarse entonces es que el "manejo" del derrame sólo cubrió la tercera parte del petróleo vertido. El resto, en un $26 \%$ está en las costas como bolas de alquitrán, enterrados bajo la arena, en los sedimentos o flotando en la superficie del océano; un $25 \%$ se calcula ya se evaporó o disolvió y $16 \%$ se dispersó de modo natural (www.restorethegulf.gov/sites/default/files/documents/pdf/OilBudgetCalc_Full_H Q-Print_111110.pdf). Por tanto, es evidente que las afectaciones se verán en su real dimensión en el futuro.

De cualquier modo, los costos inmediatamente visibles son diversos. La afectación a 445 especies de peces, 134 de pájaros, 45 de mamíferos y 32 de reptiles y anfibios, muchas en peligro de extinción como lo es el caso de la tortuga lora. El daño a las zonas costeras (más de $160 \mathrm{~km}$ ) incluyendo humedales y pantanos de Louisiana y la zona del delta del Missisipi son tal vez de lo más ilustrativo. Con ello se afectó no sólo los ecosistemas, sino también actividades productivas relacionadas a la pesca y la maricultura y que corresponden al $40 \%$ de los productos del mar que se consumen en EUA. Se suman otras afectaciones como la mencionada quema controlada de petróleo con su consecuente emisión de humo tóxico, contribuyendo a la contaminación del aire y en el calentamiento global.

Los costos por tanto son evidentes, aunque no todos visibles y medibles. Por lo pronto, BP enfrenta ya más de 42 mil solicitudes de demandas por afectación de diverso tipo, incluyendo las de estados mexicanos como Veracruz, Tamaulipas y Quintana Roo. ${ }^{5}$

Los costos del derrame han sido contabilizados sólo parcialmente pues se han expresado en términos crematísticos en el orden de 8 mil millones de dólares

\footnotetext{
${ }^{5}$ El Gobierno Mexicano ha gastado unos 30 millones de dólares en prevención y evaluación de daños a lo que se suman los gastos por venir en monitoreo. La demanda es inicialmente por 20 mil millones de dólares. Para mayores referencias sobre las actividades de monitoreo, consúltese: http://derrame.semarnat.gob.mx/
} 
con estimaciones de llegar a más de 20 mil millones. Esos montos incluyen, hasta ahora, el costo de las operaciones de contención, de perforación del pozo auxiliar, el procedimiento de sellado del pozo, la inyección de cemento , las concesiones a los estados del Golfo de México, los reclamos efectivamente pagados y los costos federales. El valor de la pérdida de biodiversidad o la afectación de ecosistemas enteros en el corto, mediano y largo plazo, entre otras cuestiones, no ha sido tomado en cuenta, proceso que además ciertamente se torna complejo pues el valor de la biodiversidad suele ser inconmensurable, razón por la que no puede medirse siempre y únicamente en términos económicos.

Es cuando menos controversial que ante este tipo de costos ocultos, el Servicio de Administración e Minerales del Departamento del Interior de EUA, adoptara en 2005 una serie de regulaciones que asumen que las propias petroleras son las que mejor pueden evaluar sus impactos ambientales (situación que ligeramente modifica, pero no resuelven, las medidas tomadas después del derrame Macondo). Y, en lo que respecta a la transportación y la necesidad de doble fondo en los barcos-cisterna (medida producto del derrame del Exxon Valdez), ésa ha sido desde entonces pospuesta, por lo pronto, al 2015. Este par de situaciones develan con nitidez el poder que tiene la industria petrolera y su lobby que mantiene como prioridad el negocio por encima de cualquier otra cuestión así sea el sustento mismo de la vida. No extraña que exista entonces una puerta giratoria entre el gobierno de EUA y la industria petrolera que permita mantener el fluido curso de sus "sucios" negocios. Así, por ejemplo, se puede identificar en el consejo asesor de BP a Leon Panetta, jefe de personal de la Casa Blanca y hoy director de la CIA; a Tom Daschle, líder de la mayoría del Senado; y a Christine Whitman de la Agencia para la Protección Ambiental. Y esto es sólo el caso de esta empresa.

Al cierre de 2010, algunos expertos reunidos en el marco de la Gulf Oil Spill Remediation Conference, aseguran que el problema en el Golfo de México sigue latente pues no es muy claro si el sellado del pozo fue completamente exitoso pero, sobre todo, porque aún hay fugas y filtraciones en un área de entre 13 y 26 km$^{2}$ alrededor del pozo (Termotto, 2010). La muerte del Golfo es inminente de seguir la tendencia pues a lo de Macondo se suman innumerables derrames, fugas y filtraciones a lo largo de la costa de estadounidense que son producto de décadas de actividad petrolera (Ibid). Por tanto, dado que lo que se juega en Macondo es en cierto modo el futuro de la extracción petrolera de aguas profundas -al menos en EUA-, no es de extrañarse que haya una fuerte manipulación por parte de autoridades y empresas involucradas sobre lo realmente sucedido, el estado de situación y futuros costos .

\subsection{Cambio climático, un reflejo de largo plazo de los costos ocultos del patrón} energético actual.

A nivel mundial, el grueso de gases de efecto invernadero son producto de la quema de combustibles fósiles asociada a la generación de electricidad y calefacción (24.6\%), así como al sector transporte (13.5\%). El cambio de uso 
de suelo (18.2\%), la agricultura (13.5\%) y la industria (10.4\%) son los siguientes rubros de relevancia, siendo la agricultura el mayor contribuyente de metano junto con algunos otros procesos industriales (Baumert et al, 2005).

En México, siguiendo las tendencias mundiales, los sectores de mayor intensidad energética son el de producción de energía (eléctrica fundamentalmente) y el de transporte. En ese mismo sentido, son ésos rubros los generadores de más gases de efecto invernadero (GEI). Los porcentajes de uso energético/generación de GEI son: transporte (18\%); generación de energía (24\%); Cambio de usos de suelo y silvicultura (14\%); desechos (10\%); procesos industriales (8\%); manufactura e industria (8\%); agricultura (7\%); emisiones fugitivas (6\%); y otros (5\%). Destacan entre los usos específicos finales, el consumo de gasolinas (16\%); la extracción y refinación de petróleo (11.6\%); producción de hierro y acero (5.15\%) y Cemento (3.6\%). En resumen se puede argumentar que en el país, la generación de energía, el transporte y los productos extractivos (incluyendo ahí parte del cambio de uso de suelo por monocultivos o deforestación) son los principales responsables de la emisiones de gases contaminantes. Rubros que, de continuar la tendencia actual, apuntan a incrementar su aporte.

Los efectos de tal dinámica nacional y mundial son múltiples, siendo el calentamiento global, de tipo antropogénico, uno de los síntomas más visibles. Producto, sobre todo, de la quema indiscriminada de combustibles fósiles, la cantidad de carbono en la atmósfera, que se mantuvo constante en los últimos 10 mil años en el rango de las 280 partes por millón (ppm), pasó a 360 ppm en 1998 y a 383 ppm en 2006 (Heinberg, 2003: 32). Esta última cifra ya es considerada por los especialistas en cambio climático, como "territorio peligroso". 6

La polarización en las contribuciones de destrucción del medio ambiente es nítidamente observable. El $20 \%$ de la población mundial que habita en países metropolitanos, ha generado el $90 \%$ de los gases de efecto invernadero en términos históricos (Godrej, 2001: 95). Más aún, la huella ecológica mundial, indicador que calcula -en base al actual modo de vida- el espacio territorial necesario, tanto para producir los recursos y energía empleados, como para asimilar los residuos generados por la humanidad, indica que ya se sobrepasa entre un $25 \%$ y un $39 \%$ al planeta Tierra, dependiendo de los cálculos. ${ }^{7}$ Necesitamos pues, en el mejor de los casos, un cuarto de planeta adicional para poder mantener los ritmos de consumo y desecho de principios del siglo $\mathrm{XXI}$; el grueso sobre todo de países desarrollados. Y es que, tan sólo el índice de emisión de $\mathrm{CO}_{2}$ ya supera los 70 millones de toneladas cada 24 horas.

Los impactos de largo plazo sólo de la acumulación de contaminantes atmosféricos están esencialmente vinculados al aumento de la temperatura y

\footnotetext{
${ }^{6}$ Así lo califica, por ejemplo, James Hansen del Instituto Goddard para el estudio del Espacio de la NASA (EUA).

7 Las estimaciones varían. Para la Global Footprint Network, la humanidad pasó de usar, en términos netos, la mitad de la biocapacidad del planeta en 1961 a 1.25 veces en 2003 (Global Footprint Network, 2004). Según Redefining Progress la biocapacidad del planeta había sido rebasada, para el año 2005, en un 39 por ciento (Venetoulis y Talbert, 2005).
} 
del nivel del mar, el incremento de eventos climáticos extremos, el cambio de los patrones de lluvia y la perdida creciente de la biodiversidad.

Así entonces y de cara a los esperados y eventuales impactos del cambio climático, es ampliamente reconocido que los países que enfrentarán los costos más altos serán aquellos cuya contribución en tanto emisiones de gases de efecto invernadero es pequeña (Bicknell, Dodman y Satterthwaite, 2009). Tales costos estarán en buena medida vinculados a riesgos actuales, dígase inundaciones, tormentas, escasez de agua, etcétera, dado que se agudizarán. A ello se suman problemas de producción de alimentos y otros impactos atípicos. Lo anterior obliga no sólo a tomar medidas para mejorar o adaptar la infraestructura, entre la que la energética es nodal, pero también evidentemente está asociado a una amplia agenda de acciones de mitigación, misma que pasa por revisar el ciclo completo de producción, distribución y consumo (inclúyase el desecho) energético-material de los espacios tanto urbanos como rurales.

El cambio climático obliga pues, a revisar seriamente cómo y en función de qué se construye el espacio-territorial y por ende, a cómo se concibe el desarrollo. La disminución, hasta cierto grado, del impacto ambiental y de la vulnerabilidad es posible, aunque algo ciertamente muy complejo y que requiere de la acción coordinada de diversos actores, especialmente de gobierno en tanto responsables de la política pública y de la sociedad en general, en cuanto que puede construir una articulación suficiente como para presionar para la toma de acciones en uno u otro sentido.

\section{Securitización de los recursos naturales: resistencia a un cambio de paradigma.}

Las agendas que securitizan los recursos naturales estratégicos (petróleo y ciertos minerales, sobre todo) y los entornos naturales donde se encuentran, son producto de construcciones subjetivas, propias del Estado y de las clases que lo detentan, sobre el riesgo y la incertidumbre. Así, mientras la securitización de los recursos energéticos es bien conocida, siendo la invasión a Irak una de las expresiones más recientes y crudas de la misma, la securitización ambiental es más reciente. Ésa gira entorno a cuatro elementos: 1) el aumento de la población; 2) la accesibilidad a recursos naturales clave; 3) la destrucción del medio ambiente; y 4) el cambio climático.

Nótese entonces que la agenda de seguridad energética depende en cierto grado, pero también modela en buena medida la agenda de seguridad ambiental. Esto es, son sinérgicas. Ello puesto que la producción de energía fósil destruye ecosistemas, su quema genera gases de efecto invernadero que producen cambio climático y a su vez altera ecosistemas y los ciclos biogeoquímicos del planeta. Tal destrucción y cambio climático limitan, en principio, la propia producción y quema de combustibles fósiles indiscriminada, pero también la de otros recursos como los mineros. Al mismo tiempo, lastima la capacidad productiva de alimentos y de acceso a agua fresca de buena calidad, pero aún más, pone en entre dicho el futuro mismo de la humanidad, al 
menos tal y como la conocemos pues compromete la vida misma en el planeta. Es en este sentido que se apunta a la necesidad de hablar de una seguridad humana.

La securitización de estos aspectos, lo energético, ambiental y humano es una cuestión delicada pues parte de la noción tradicional de seguridad, esto es, una noción construida desde y para el Estado que eventualmente podría requerir de la intervención de la fuerza "legítima" del Estado según sea el grado del riesgo e incertidumbre.

En este sentido, las fuerzas militares ya han jugado y siguen jugando un papel clave para asegurar el acceso, gestión y usufructo de tales recursos energéticos a escala planetaria manteniendo el actual curso de los negocios (business as usual). Así, los militares ponen condiciones o facilitan tales actividades a favor de ciertos actores empresariales pero bajo el velo del "libre mercado". Esto es, a decir de Saxe Fernández (2003), la operación coordinada de "la mano invisible del mercado" y de la mano visible del Pentágono.

Y es que geopolítica del petróleo es clara y se aprecia mejor si se toma nota de

\begin{tabular}{|c|c|c|c|c|c|}
\hline $\begin{array}{r}\text { Cuadro } 1 \\
\text { Princi } \\
\text { im } \\
\text { hidroca }\end{array}$ & $\begin{array}{l}\text { les } \\
\text { orta } \\
\text { purc }\end{array}$ & $\begin{array}{l}\text { roductores } \\
\text { ores de pet } \\
-2008 \text { (tor }\end{array}$ & $\begin{array}{l}\text { óle } \\
\text { ela }\end{array}$ & $\begin{array}{l}\text { ortadores } \\
\text { y otros } \\
\text { s métricas }\end{array}$ & \\
\hline PRODUCTOF & & EXPORTADO & RES & IMPORTADC & RES \\
\hline Rusia & 494 & $\begin{array}{l}\text { Arabia } \\
\text { Saudita }\end{array}$ & 355 & EUA & 564 \\
\hline $\begin{array}{l}\text { Arabia } \\
\text { Saudita }\end{array}$ & 452 & Rusia & 241 & Japón & 199 \\
\hline EUA & 320 & Irán & 120 & China & 175 \\
\hline Irán & 206 & $\begin{array}{l}\text { Emiratos } \\
\text { Árabes }\end{array}$ & 108 & India & 128 \\
\hline China & 194 & Nigeria & 102 & Corea & 116 \\
\hline Canadá & 152 & Angola & 92 & Alemania & 105 \\
\hline México & 146 & Noruega & 90 & Italia & 88 \\
\hline Venezuela & 126 & Kuwait & 89 & Francia & 83 \\
\hline Kuwait & 124 & Irak & 88 & España & 61 \\
\hline $\begin{array}{l}\text { Emiratos } \\
\text { Árabes }\end{array}$ & 120 & Venezuela & 74 & Holanda & 57 \\
\hline
\end{tabular}
que a nivel mundial, mientras los mayores productores de petróleo son Rusia, Arabia Saudita, EUA, Irán, China, Canadá, México, Venezuela, Kuwait y Emiratos Árabes Unidos; los principales importadores netos son EUA, Japón, China, India, Corea del Sur, Alemania, Italia, Francia, España y Holanda.

EUA es el mayor importador mundial de petróleo y por mucho pues acapara el equivalente al total las importaciones de Japón, China, India y España en conjunto (AIE, 2010: 11). Véase cuadro 1. De ahí que ese país esté dispuesto a asumir mayores riesgos socioambientales vinculados al mantenimiento del patrón energético fósil (por eso su legislación laxa en regulación de la industria petrolera o en materia de cambio climático) y desde luego también se explica el gran rol de esa potencia en lo que se ha calificado como guerras por los recursos (Klare, 2001); un panorama que se agudiza cada vez más conforme lo hace el peak oil, los patrones de consumo y el cambio climático.

A contracorriente de la securitización o geopolitización de los recursos, vale hablar de una seguridad ecológica, o aún más, de una seguridad amplia, 
integral y social -o lo que Oswald (2010) denomina como seguridad HUGE-, entendida ésa no desde la visión del estado y los intereses de grupo que suele representar, sino desde los intereses y necesidades sociales del grueso de la población (incluyendo cuestiones de género, diversidad cultural, valores históricos y tradicionales, legado generacional, etcétera).

En esta coyuntura, es muy importante tener en cuenta que la securitización de lo energético y lo ambiental pasa por el control concreto de los territorios por lo que es clave la aceptación o subordinación de los pueblos. Ello suele generar y/o intensificar descontentos sociales y pone en riesgo los intereses nacionales de largo plazo pues somete territorios concretos a las dinámicas e intereses de ciertos actores, muchos de los cuales suelen ser foráneos (por la vía, por ejemplo, de la IED en proyectos extractivos). Las implicaciones, no obstante, no sólo son a escala de potenciales conflictos entre naciones, sino sobre todo a nivel interno. En particular me refiero a los conflictos distributivos. Ésos pueden adquirir la forma de:

- disputas originadas por la degradación o disminución del recurso (o por desastres naturales);

- disputas por el acceso, uso y usufructo de los recursos; o

- conflictos derivados de esquemas de acumulación por desposesión ${ }^{8}$ que privan el entorno natural como medio de vida, sea por la vía de la privatización y extranjerización formal de los recursos, o debido a la pérdida del entorno como consecuencia de su explotación irracional o por desastres provocados (como los derrames petroleros).

En ese panorama debe precisarse entonces que el control de los territorios en $\mathrm{AL}$ en el contexto actual ha sido violento y la modalidad preferida es la de ocupación estratégica, la guerra de baja intensidad y la criminalización de la resistencia social. Ello ha incluido al sector petrolero y minero, así como a otros sectores como el maderero, el del negocio del agua, etcétera. Se trata de un panorama en el que la "transformación" y "modernización" de las fuerzas armadas y policíacas para asumir el orden interno es clave para cualquier intento de control y apertura de espacios territoriales estratégicos al mercado. En lo concreto ello muchas veces también significa establecer condiciones sociales, ambientales y políticas ideales para atraer la inversión extranjera directa en actividades netamente extractivas, típicamente de nulo o bajo encadenamiento productivo endógeno.

El modelo primario-exportador (o extractivista) estimulado por IED, perdidamente se asume por parte de la elite dirigente y gobernante (Domhoff, 1969) latinoamericana como motor de la economía (a la par de ciertos y tecnológicamente bien controlados procesos de manufactura de tipo maquilador). Lo anterior requiere por tanto, además de asumir los costos ambientales ocultos asociados a tales actividades, que los gobiernos crean condiciones favorables a la inversión, por ejemplo al fortalecer el estado de derecho de tal suerte que se de seguridad a las inversiones, al dar garantías a la inversión foránea, al disminuir los aranceles e impuestos a los grandes

\footnotetext{
${ }^{8}$ Sobre una teorización de los conflictos ambientales distributivos, léase: Martínez-Alier, 2006. Sobre la conceptualización de "acumulación por desposesión": Harvey, 2004.
} 
capitales, al adoptar legislaciones y normas socio-ambientales flexibles y "competitivas", y al despejar lo más posible los "inconvenientes" sociales.

El Departamento Nacional de Planeación de Colombia asume tal noción al precisar que: “...la seguridad estimula la inversión y ésta, con responsabilidad social, permite avanzar en la superación de la pobreza y la construcción de equidad". Y especifica prioridades a partir de lo que la Escuela Superior de Guerra (2009) denomina como el "circulo virtuoso de la seguridad": 1) Inversión y seguridad; 2) confianza y estabilidad; 3) inversión privada; 4) crecimiento económico; 5) impuestos e inversión social; 6) bienestar social y satisfacción de necesidades. Desde dicha óptica, se considera entonces que un orden seguro es un orden "democrático" capaz de garantizar la estabilidad del mercado (Loveman, 2006), y es el mercado, desde luego, el mejor ente para distribuir la riqueza y satisfacer las necesidades sociales. El gobierno de México actual considera como válido ese mismo modelo (léase: Delgado y Romano, 2010). La interpretación no puede ser más errónea y lo comprueba la propia historia del capitalismo y en especial la de los países periféricos.

Por lo anterior, es pertinente definir claramente el papel de las fuerzas de militares y de seguridad. La propuesta predominante para los países latinoamericanos, por ejemplo, ha sido que ésas sean funcionales a proyectos extractivistas y desnacionalizadores de la riqueza nacional a través de asegurar los recursos naturales desde una visión tradicional y por tanto desde los intereses de los grupos de poder y ciertamente no los de los pueblos. Con ello se consolida que sean los pueblos, los más pobres, los que asuman en lo concreto el grueso de la deuda ecológica que los países ricos tienen con la región y con el resto de la periferia. De ahí que se observa la necesidad de instituir un esquema basado en la justicia socio-ambiental y que podría ser el de la "seguridad ecológica" o, aún más, el de una "seguridad amplia, integral y social" como fundamento de la soberanía y seguridad nacional de las naciones latinoamericanas y sus pueblos.

\section{Reflexión final. Repensando el desarrollo como fundamento de cambio de paradigma.}

El patrón energético es fundamental en la economía mundial. El mantenimiento del patrón de combustibles fósiles hace posible no sólo la modalidad actual de producción-circulación-consumo sino la propia dinámica y dimensión de la acumulación de capital alcanzada a principios del siglo XXI.

El típico entendimiento de que el desarrollo tiene como fundamento el crecimiento económico, o peor aún que ése es el sinónimo segundo, dibuja un esquema que apunta, mas temprano que tarde, a la debacle socio-ambiental puesto que el crecimiento económico obligadamente requiere de la transformación de la naturaleza hacia un estado de mayor de baja, es decir, en desechos, y dado que esa transformación es irrevocable, el medio ambiente establece límites al subsistema económico. Para Georgescu-Roegen (1971: 67), el dilema es claro: “... no es preciso disponer de argumentos sofisticados para ver que el máximo de cantidad de vida exige una tasa mínima de 
agotamiento de los recursos naturales [...] Todo uso de los recursos naturales para satisfacer necesidades no vitales lleva consigo una menor cantidad de vida en el futuro".

En tal sentido, repensar el desarrollo es clave para la construcción de alternativas para la vida. De entrada desligarlo del crecimiento económico es fundamental para luego asociarlo a un decrecimiento sustentable que puede ser definido como, “....una reducción equitativa de la producción y el consumo que incrementa el bienestar humano y mejora las condiciones ecológicas al nivel local y global, en el corto y largo plazo" (Schneider et al, 2010). El decrecimiento sustentable no es pues aquel resultante de la recesión 0 depresión económica ni del deterioro de las condiciones sociales, sino esencialmente de la reducción de los flujos de materiales y de energía que sostienen a la economía.

Pero el decrecimiento sustentable y el desarrollo puede y debería tomar múltiples versiones teniendo en común su móvil central: construirse en armonía con la naturaleza y desde la perspectiva de la vida de todos y cada uno de los individuos (esto es que se visualiza desde la unidad ser humano-naturaleza); que considera la complejidad de los contextos de cada espacio o región y; que aprovecha y conserva la diversidad y riqueza cultural y de conocimientos ahí existentes.

En buena medida esto implicará no sólo reducir los consumos despilfarradores de las clases acomodadas, tanto en los países centrales como periféricos, sino modificar todo el proceso y formas de producción, circulación y consumo que externalizan los costos ambientales hipotecando el futuro a favor del presente. Para el caso específico de los países periféricos como los latinoamericanos, será esencial buscar las modalidades para atender las apremiantes necesidades sociales y que requerirán, en un principio, un aumento del flujo energético-material pero que se genera desde otra perspectiva y modalidad y para otra finalidad distinta, esto es para otras formas de desarrollo. Esto significa que el desarrollo se vincula al "buen vivir", noción que variará para cada sociedad pero que incluye en cualquiera de sus versiones no sólo lo material sino lo emocional, lo intelectual y lo espiritual. Si el desarrollo no requiere del crecimiento económico per se -pero sí de economía- y además incluye otras variables, entonces las actividades extractivistas, primarioexportadoras, que devastan el medio ambiente y sobre-explotan al ser humano no tienen razón de ser. Otras modalidades de desarrollo deben por tanto partir de la noción de justicia socio-ambiental, de evitar la deuda ecológica y el comercio socio-ecológicamente desigual, de disminuir los conflictos ecológicos distributivos y, en general, de reducir los flujos de materiales y de energía de la economía al tiempo que aumenta la calidad de vida y toma en cuenta valores no crematísticos y servicios recíprocos no mercantilizados.

En materia específicamente energética se tiene que apostar hacia la transición del paradigma energético imperante hacia uno cada vez más soportado por flujos de energía y no stocks de energía. La apuesta por energías alternativas, menos agresivas al medio ambiente en todo su ciclo de vida o in toto, no será viable sino es acompañado por una disminución de los patrones de consumo 
energético y un acceso descentralizado y justo a la energía. El proceso de transición requerirá de mucha energía, y el grueso será en un principio fósil. Por ello que el actual despilfarro es doblemente cuestionable.

Estrategias como las denominadas de "emisiones netas evitadas" (propuestas por Ecuador en el marco de la reunión de las partes sobre cambio climático COP16, por ejemplo, desde su proyecto Yasuní-ITT ${ }^{9}$ ) son un paso pero no ninguna solución a fondo. Un retroceso son aquellas que abogan por un capitalismo verde pues no buscan un cambio real de paradigma, dígase el de asegurar la vida tal y como la conocemos, sino en primer lugar mantener el sistema de producción, circulación y consumo imperante con todas sus relaciones asimétricas de poder, al tiempo que se apuntala como mecanismo anticrisis, es decir, como un nicho más acumulación de capital.

A estas alturas debería ya estar bien claro que el crecimiento económico sostenido no puede mantenerse al infinito en un planeta finito. Desarrollo sustentable en su significado positivo no significa cero impacto ambiental, sino un impacto lo menos dañino posible en el corto, mediano y largo plazo; de ahí la propuesta de decrecimiento sustentable.

El presidente Evo Morales de Bolivia tiene razón en afirmar que la disyuntiva hoy por hoy está entre "planeta o muerte" y en dar cuenta que las alternativas deberán construirse en colectivo y para lo cual se requiere, entre muchos factores, "... hacer juntos una nueva tesis [o muchas tesis] por la vida, por las generaciones actuales y futuras."

\section{Bibliografía}

- AIE - Agencia Internacional de Energía (2010). Key World Energy Statistics 2010. Paris, Francia.

- Bank Information Center et al (2006). How the World Bank's Energy Framework Sells the Climate and Poor People Short. EUA, septiembre.

- Baumert, Kevin., Herzog, Timothy., y Pershing, Jonathan (2005). Navigating the numbers. Green gas data and International Climate Policy. World Resources Institute. EUA.

- Bicknell, Jane, Dodman, David y Satterthwaite, David (2009). Adapting Cities to Climate Change. Earthscan. Londres/Washington.

- Cambell, Colin (1997). The Coming Oil Crisis. Multi-Science and Petroconsultants. EUA.

- Deffeyes, Kenneth (2001). Hubbert's Peak: The Impeding World Oil Shortage. Princenton University Press. EUA.

- Delgado Ramos, Gian Carlo (2008). Sin Energía. Cambio de paradigma, retos y resistencias. Plaza y Valdés. México.

\footnotetext{
${ }^{9}$ Consiste en mantener inexploradas las reservas de petróleo pesado ubicadas en el campo ITT (Ishpingo, Tiputini y Tamcococha) en el Parque Nacional Yasuní. A cambio el gobierno propone una retribución de al menos 3,600 mdd, equivalentes al $50 \%$ de los recursos que Ecuador recibiría si optara por explotar dichas reservas. Para mayores referencias, véase: http://yasuni-itt.gob.ec
} 
- Delgado-Ramos, Gian Carlo y Romano, Silvina (2010). "Economía política de la seguridad interna en América Latina: transferencia de excedentes, 'narco-insurgencia' y control social." Revista Espacio Crítico. No. 13. Pp. 28 - 45. Colombia, Julio - Diciembre.

- Domhoff, William (1969). ¿Quién gobierna Estados Unidos? Siglo XXI. México.

- Epstein, Paul R. y Selber, Jesse (eds) (2002). A life cycle analysis of its health and environmental impacts. The Center for Health and the Global Environment. Harvard Medial School. EUA.

- Escuela Superior de Guerra (2009). "La política de consolidación de la seguridad democrática y los recursos para el sostenimiento de las Fuerzas Armadas". Departamento Nacional de Planeación. Escuela Superior de Guerra. Colombia.

- Georgescu-Roegen, Nicholas (1971). The entropy law and the economic process. Harvard University Press. EUA.

- Godrej, Dinyar (2001). No-Nonsense Guide to Climate Change. Verso. Reino Unido.

- Harvey, David (2004). El Nuevo Imperialismo. Akal Ediciones. España.

- Heinberg, Richard (2003). The Party's Over. Oil, war and the fate of industrial societies. New Society Publishers. Canadá.

- Homer-Dixon, Thomas (2007). The Upside of Down. Vintage. Canadá.

- Global Footprint Network (2004). Humanity's Footprint 1961-2003. EUA.

- Klare, Michael (2001). Resource Wars: the new landscape of global conflict. Metropolitan / Owl Book. Nueva York, EUA:

- Loveman, Bryan (comp) (2006) Addicted to Failure. US Security Policy in Latin America and the Andean Region. Maryland: Rowman \& Littlefield.

- Martínez-Alier, Joan (2006). El Ecologismo de los Pobres. Conflictos ambientales y lenguajes de valoración. Icaria. España.

- Oswald Spring, Úrsula (2010). Cambio climático, conflictos sobre recursos y vulnerabilidad social en: Delgado, Gian Carlo., Gay Carlos., Imaz, Mireya., y Martínez, Amparo. México frente al cambio climático. Retos y oportunidades. CCA-CEIICH-PUMA-PCC, UNAM. México.

- Saxe-Fernández, John (2003). "Irak, México y la mano invisible." La Jornada. México, 6 de marzo de 2003.

- Schneider, Francois., Kallis, Giorgios., Martínez-Alier, Joan (2010). "Crisis or oportunity? Economic degrowth for social equity and ecological sustainability. Introduction to this special issue." Journal of Cleaner Production. No. 18. Elsevier. Amsterdam, Holanda.

- Steffes, Dale W. "A proposed world oil stability policy" en, The World Oil \& Gas Industries in the 21st Century: Conference Proceedings of the 16th Annual North American Conference of the IAEE. EUA, 1994: 20-29

- Termotto, Tom (2010). "The Gulf of Mexico is Dying". Phoenix Rising from the Gulf. EUA, 1 de diciembre. En: http://phoenixrisingfromthegulf.wordpress.com/2010/12/01/the-gulf-ofmexico-is-dying/ Consultado el 30 de diciembre de 2010.

- Venetoulis, Jason y Talberth, John (2005). Ecological Footprint of Nations, Update 2005. Redefining Progress. EUA. 